\title{
A JOINT STOCK AND BOND MARKET BASED ON THE HYPERBOLIC GAUSSIAN MODEL
}

\author{
NICOLE BÄUERLE* AND ROBIN PFEIFFER*
}

\begin{abstract}
In this paper, we introduce a joint bond and stock market model based on the state price density approach as a mean to discount future payments - whether these are stochastic dividend payments or secure repayments of government zerobonds. Based upon a recipe of Rogers (1997), we define a state price density model, the so-called Hyperbolic Gaussian model which allows for closed form zerobond prices and stock prices in an arbitrage-free way. It is particularly useful for insurance applications where large time horizons are considered. We estimate the joint factor model using the extended Kalman filter. The model we propose here is computationally much simpler than other models which have been considered in the literature.
\end{abstract}

KEY WORDS: Interest rate models, Potential approach, Dividend discount approach, Kalman filter, Monte Carlo simulation. JEL SubJeCt CLAssifications: G12, C51, E43, G22

\section{INTRODUCTION}

In order to compare stochastic cash flows, they have to be discounted which requires a stochastic term structure model. Most term structure models in the literature have been developed for banking applications (for an overview see e.g. Filipovic (2009), Cairns (2008), Musiela \& Rutkowski (2005), Rebonato (2002) or Brigo \& Mercurio (2001)). For insurance companies term structure dynamics play a central role. What is special in insurance applications is that first, the duration of products (like life or pension insurance contracts) typically exceed the available maturities of the currently observable term structure. Next, the insurer requires a term structure model to discount his contractual liabilities which typically requires Monte Carlo simulation due to the complexity. Finally life and pension insurers at least in continental Europe mainly invest in fixed income securities. Future payoffs of insurance contracts therefore depend on intermediate returns achieved in fixed income markets and intermediate portfolio allocation which yields path-dependence for most insurance applications.

In this paper we consider the so-called Hyperbolic Gaussian model which is a special case of the state price density models introduced in Rogers (1997). The state price density $\left(\varsigma_{t}\right)$ is sometimes called risk-neutral density or state-price deflator and it can be used to price contingent claims (see e.g. Duffie (1992)). If $C_{T}$ is the payoff of a contingent claim at time $T$, then its price at time $t$ can be defined by

$$
C_{t}=\frac{\mathbb{E}\left[\varsigma_{T} C_{T} \mid \mathcal{F}_{t}\right]}{\varsigma_{t}}
$$

In the classical Black-Scholes model with one stock and constant shortrate $r$, drift $\mu$ and volatility $\sigma$, the state price density is for example given by $\varsigma_{t}=L_{t} B_{t}^{-1}$ where $B_{t}=e^{r t}$ and

$$
L_{t}=\exp \left(-\frac{1}{2}\left(\frac{\mu-r}{\sigma}\right)^{2} t-\left(\frac{\mu-r}{\sigma}\right) Z_{t}\right) .
$$

* Karlsruhe Institute of Technology (KIT), Institute for Stochastics, Kaiserstr. 89, 76133 Karlsruhe, Germany nicole.baeuerle@kit.edu and robin.pfeiffer@kit.edu. Phone: +49-721-608-48152. Fax: +49-721-608-46066.

The underlying project is funded by the Bundesministerium für Bildung und Forschung of Germany under promotional reference 03BAPAD2. The authors are responsible for the content of this article. 
The process $\left(Z_{t}\right)$ is a Brownian motion and $L_{T}$ can be used to define the risk neutral measure $\frac{d \mathbb{Q}}{d \mathbb{P}}=L_{T}$. The price $C_{t}$ at time $t$ of a contingent claim which pays $C_{T}$ at time $T$ is given by

$$
C_{t}=B_{t} \mathbb{E}_{\mathbb{Q}}\left[C_{T} B_{T}^{-1} \mid \mathcal{F}_{t}\right]=\frac{1}{L_{t} B_{t}^{-1}} \mathbb{E}\left[C_{T} L_{T} B_{T}^{-1} \mid \mathcal{F}_{t}\right]=\frac{\mathbb{E}\left[C_{T} \varsigma_{T} \mid \mathcal{F}_{t}\right]}{\varsigma_{t}}
$$

where we used the Bayes rule for the second equation. In case of a default free bond we have $C_{T}=1$ and an explicit bond price formula essentially requires that $\mathbb{E}\left[\varsigma_{T} \mid \mathcal{F}_{t}\right]$ can be computed.

One representative of this family is the model considered in Cairns (2004) (see also Cairns (2008)). There

$$
\varsigma_{t}:=\int_{t}^{\infty} \phi_{s} M(t, s) d s
$$

where $\phi$ is a deterministic function and $(M(t, s))$ for $t \leq s \leq \infty$ is a family of strictly positive diffusion martingales. This model is also a special case of the Flesaker and Hughston framework (Flesaker \& Hughston (1996)), indeed this point of view is taken in Cairns (2004). For special choices of $\phi$ and $M$ (in particular $M$ is taken as a function of an Ornstein-Uhlenbeck state process), Cairns shows some properties of this model and in particular recommends it for longterm interest rate modeling, since it provides sustained periods of both high and low interest rates. This is of particular importance in insurance applications. Moreover, it guarantees positive interest rates. The price of a bond at time $t$ with maturity $T$ in Cairns' model is given by

$$
P(t, T)=\frac{\int_{T}^{\infty} M(t, s) \phi(s) d s}{\int_{t}^{\infty} M(t, s) \phi(s) d s} .
$$

Since the price depends on the whole path of $M(t, s)$ this formula is computationally demanding and slow in Monte Carlo simulations.

The main aim of our paper is to present the Hyperbolic Gaussian model as a model with similar features but one which is very easy to implement and thus interesting for practical purposes. We also show that the Hyperbolic Gaussian model can be extended to a joint bond and stock market - a feature which is only theoretically possible in Cairns' model. Indeed the advantage of an explicit model for the state price density, like the Hyperbolic Gaussian model, lies in avoiding the integral over the stochastic shortrate in discounting functions. This is particularly helpful in the pricing of complex securities such as in life insurance, which typically require Monte Carlo simulation. In that case, when e.g. a shortrate model is used, discounting based on shortrate integrals are necessarily path dependent and the frequency of intermediate steps is determined by the shortrate integral and hence ultimately the discounting function, not the security to be priced and its cash flow. Using an explicit model for the state price density implies that intermediate simulation points are determined by the security to be priced only and hence typically far less random variables have to be used in Monte Carlo simulations based upon the state price density. Thus, we consider here the Hyperbolic Gaussian model which on the negative side does not guarantee positive interest rates, however is computationally much more efficient than the model in Cairns (2004) and shows a similar behavior. Indeed, in both models when implemented as a two-factor model, one state vector component usually coincides with the slope of the interest curve whereas the other component coincides with the level. For a detailed comparison of both models, including parameter estimation results and properties see Pfeiffer (2010) (cp. also Section 4.3). Moreover, what is important for us, is that the Hyperbolic Gaussian model can produce longer periods of low interest rates and the historical fit using the extended Kalman filter is very good (see Section 4). Thus we think this model might be helpful for simulation tools like DFA (dynamic financial analysis) in insurance companies. Let us note here that also in Yao (2001) an exponential state price density has been used to derive bond prices, option prices and foreign exchange rates.

Since pure term structure models are insufficient to simulate the asset side of an insurance company, a major task is to extend the bond market consistently. Wilkie (1984, 1986) argues that an insurance or pension fund model should at least incorporate consol yields, stock prices, 
dividend yield and inflation. In Wilkie (1995) the investment model is even expanded. In order to incorporate at least a stock we use two approaches: A dividend discount approach and an extension of the Black Scholes model. In the dividend discount approach, the stock price at time $t$ is interpreted as the value of all future dividends discounted at time $t$. Such an approach easily fits into the state price density framework which is used to discount dividends. In a continuous dividend paying setting this has been used in Graziano \& Rogers (2006). In the model investigated in Cairns (2004) such an approach is also possible from a theoretical point of view however practically unfeasible due to computational limitations. For the Hyperbolic Gaussian model on the other side it provides an arbitrage-free, implementable stock pricing framework.

The outline of our paper is as follows: In the next section we review the state price density approach and present the Hyperbolic Gaussian model. It is based upon multidimensional Ornstein-Uhlenbeck state processes. The special one-dimensional case can already be found as an example in Rogers (1997) and Cairns (2008). Explicit bond-price formulas are derived as well as zerobond rates and the shortrate. It is also shown that the family of bond prices is free of arbitrage. Section 3 then extends this model to an arbitrage-free joint bond and stock market model by using the dividend discount approach and an extension of the Black Scholes model. Section 4 is dedicated to estimating the model parameters. Since we have a factor model we use the extended Kalman filter for estimation of the model parameters in a Quasi-MaximumLikelihood approach. Only the implementation for the dividend discount approach is explained. The unobservable process is the state process and the observable process is the term structure. Since the measurement equation is nonlinear we use the extended Kalman filter. The procedure is applied to a three-dimensional factor process and one stock using a dataset of the Federal Reserve consisting of yields derived from the US Treasury securities and the S\&P500. The estimation results and possible problems are discussed. A short conclusion and an appendix containing the proofs of some of the theorems in Section 2.2 completes the paper.

\section{The Extended Rogers Framework and the Hyperbolic Gaussian Model}

2.1. The Extended Rogers Framework. Rogers (1997) presented a generic approach to model the state price density as a function of an underlying Markovian state vector process. More precisely, one has to choose a continuous-time Markov process $\left(X_{t}\right)$ with values in $\mathbb{R}^{d}$ and a positive function $f$ with domain $\mathbb{R}^{d}$, which, together with a parameter $\alpha \in \mathbb{R}$ provides the state price density process $\left(\varsigma_{t}\right)$ by

$$
\varsigma_{t}=e^{-\alpha t} f\left(X_{t}\right), \quad t \geq 0 .
$$

We assume that all random variables are defined on a common probability space $(\Omega, \mathbb{F}, \mathbb{P})$ where $\mathbb{P}$ is the so-called pricing measure. The process $\left(X_{t}\right)$ is often called state process. In what follows let $\left(\mathcal{F}_{t}\right)$ be the filtration generated by $\left(X_{t}\right)$, i.e. $\mathcal{F}_{t}=\sigma\left(X_{s}, s \leq t\right)$. We will always assume that the expectation of $f\left(X_{t}\right)$ exists. Using the definition of $\varsigma_{t}$, the current price $C_{t}$ of a contingent claim at time $t$ which pays $C_{T}$ at time $T>t$ under the state price density approach is given by

$$
C_{t}=\frac{\mathbb{E}\left[\varsigma_{T} C_{T} \mid \mathcal{F}_{t}\right]}{\varsigma_{t}}=e^{-\alpha(T-t)} \frac{\mathbb{E}\left[f\left(X_{T}\right) C_{T} \mid X_{t}\right]}{f\left(X_{t}\right)}
$$

This provides a formula which can conveniently be evaluated by Monte Carlo methods since only the conditional distribution of $X_{T} \mid X_{t}$ has to be simulated. This is in contrast to shortrate models where usually the whole shortrate path $\left(r_{t}\right)$ has to be simulated to obtain the discount function $\int_{t}^{T} r_{s} d s$. The price of a zerobond with maturity at time $T$ is therefore given by

$$
P(t, T)=e^{-\alpha(T-t)} \frac{\mathbb{E}\left[f\left(X_{T}\right) \mid X_{t}\right]}{f\left(X_{t}\right)}
$$

and hence a measurable function of the underlying state $X_{t}$. The question now is how to choose the function $f$ and the state vector dynamics. Several important theoretical and practical properties restrict our choices. First, a basic requirement for applicability is that zerobond 
prices - and hence interest rates - are available in closed form. Given (2.2), this implies that $\mathbb{E}\left[f\left(X_{T}\right) \mid X_{t}\right]$ must be available in closed form. Yet another important requirement is that interest rates are mean reverting in the sense that we want historically observed interest rates to occur repeatedly in simulations. Following Litterman \& Scheinkman (1991), we know that to realistically model term structure dynamics, multi-dimensional state processes $\left(X_{t}\right)$ are required. Typical choices for $\left(X_{t}\right)$ therefore are processes whose components are Ornstein-Uhlenbeck or Cox-Ingersoll-Ross processes. The following theorems derive further important conditions. The first statement can be found in Rutkowski (1997), Section 3.

Theorem 2.1. If the state price density is a supermartingale, then the term structure model is free of arbitrage.

The next theorem links non-negativity of interest rates with the state price density (cf. also Cairns (2008), Theorem 8.2).

Theorem 2.2. Interest rates are non-negative if and only if the state price density is a supermartingale.

Proof. Interest rates are non-negative if and only if $1 \geq P(t, T)$ holds for all $t \in[0, T]$, hence

$$
1 \geq P(t, T)=\frac{\mathbb{E}\left[\varsigma_{T} \mid X_{t}\right]}{\varsigma_{t}}
$$

This is equivalent to $\varsigma_{t} \geq \mathbb{E}\left[\varsigma_{T} \mid X_{t}\right]$ and hence to $\left(\varsigma_{t}\right)$ being an $\left(\mathcal{F}_{t}\right)$-supermartingale.

Note, though, that in Theorem 2.1 the supermartingale property of the state price density is only a sufficient condition for obtaining an arbitrage-free market, the condition in Theorem 2.2 is necessary. If we give up the supermartingale property of $\left(\varsigma_{t}\right)$, all that is required for a term structure model is a multi-dimensional mean reverting process and a positive function $f$ which guarantee that $\mathbb{E}\left[f\left(X_{T}\right) \mid X_{t}\right]$ is available in closed form. If no-arbitrage can be shown, we have a viable term structure model, albeit one which allows for negative interest rates as many currently used term structure models.

2.2. The Hyperbolic Gaussian model. In this section, we will present an arbitrage-free term structure model which we call Hyperbolic Gaussian model in which the state price density is not a supermartingale and hence no-arbitrage has to be shown separately.

The Hyperbolic Gaussian model is the fourth example of Rogers (1997), specified by the choice of the function $f(x)=\cosh \left(\gamma^{\prime} x+c\right)$ where $\gamma \in \mathbb{R}^{d}, c \in \mathbb{R}$ and the state process $\left(X_{t}\right)$ which is given by the follow dynamics

$$
d X_{t}=\kappa\left(\tilde{\mu}-X_{t}\right) d t+C d Z_{t}
$$

where $\kappa=\operatorname{diag}\left(\kappa_{i}\right) \in \mathbb{R}^{d \times d}$ is a diagonal matrix with $\kappa_{1}, \ldots, \kappa_{d}$ on the diagonal, $\tilde{\mu} \in \mathbb{R}^{d}, C$ is a $d \times n$ matrix and $Z_{t}=\left(Z_{t}^{1}, \ldots, Z_{t}^{n}\right)$ is an $n$-dimensional Brownian motion under the pricing measure with instantaneous correlation matrix $C C^{\prime}=: \rho=\left(\rho_{i j}\right)$. The state process therefore is an Ornstein-Uhlenbeck process under the pricing measure. Hence the state price density formula yields

$$
\varsigma_{t}:=e^{-\alpha t} \cosh \left(\gamma^{\prime} X_{t}+c\right)=\frac{1}{2} e^{-\alpha t}\left(\exp \left(\gamma^{\prime} X_{t}+c\right)+\exp \left(-\gamma^{\prime} X_{t}-c\right)\right)
$$

To simplify notation, we introduce the real-valued process $V_{t}:=\gamma^{\prime} X_{t}$. From (2.3) it follows that

$$
d V_{t}=\gamma^{\prime} \kappa\left(\tilde{\mu}-X_{t}\right) d t+\hat{C} d Z_{t}
$$

where $\hat{C}:=\gamma^{\prime} C$. Hence we can express the state price density as

$$
\varsigma_{t}:=e^{-\alpha t} \cosh \left(V_{t}+c\right)=\frac{1}{2} e^{-\alpha t}\left(\exp \left(V_{t}+c\right)+\exp \left(-V_{t}-c\right)\right) .
$$


An important property of Ornstein-Uhlenbeck processes is that the conditional distribution of $X_{T}$ given $X_{t}$ is (multivariate) normal with

$$
\mathbb{E}\left[X_{T} \mid X_{t}\right]=e^{-\kappa(T-t)} X_{t}+\left(1-e^{-\kappa(T-t)}\right) \tilde{\mu}
$$

whereby $e^{-\kappa(T-t)}:=\operatorname{diag}\left(e^{-\kappa_{i}(T-t)}\right) \in \mathbb{R}^{d \times d}, 1-e^{-\kappa(T-t)}:=\operatorname{diag}\left(1-e^{-\kappa_{i}(T-t)}\right) \in \mathbb{R}^{d \times d}$ and conditional covariance matrix

$$
\Sigma(t, T):=\operatorname{Cov}\left[X_{T} \mid X_{t}\right]=\left(\frac{\rho_{l k}}{\kappa_{k}+\kappa_{l}}\left(1-e^{-\left(\kappa_{k}+\kappa_{l}\right)(T-t)}\right)\right)_{l, k=1, \ldots, d} .
$$

Theorem 2.3. For the Hyperbolic Gaussian model as defined above, the price of a zerobond at time $t$ with maturity $T$ is

$$
P(t, T)=e^{-\alpha(T-t)} \frac{\cosh \left(\mathbb{E}\left[V_{T} \mid X_{t}\right]+c\right)}{\cosh \left(V_{t}+c\right)} e^{\frac{1}{2} \gamma^{\prime} \Sigma(t, T) \gamma} .
$$

Proof. Using (2.1) with $C(T)=1$, the bond price is given by

$$
P(t, T)=e^{-\alpha(T-t)} \frac{\mathbb{E}\left[f\left(X_{T}\right) \mid X_{t}\right]}{f\left(X_{t}\right)}
$$

with $f\left(X_{t}\right)=\cosh \left(\gamma^{\prime} X_{t}+c\right)=\cosh \left(V_{t}+c\right)$. Using the fact that $X_{T} \mid X_{t}$ has a (conditional) multivariate normal distribution, we get

$$
\begin{aligned}
\mathbb{E}\left[\exp \left(V_{T}+c\right) \mid X_{t}\right] & =\exp \left(\mathbb{E}\left[V_{T}+c \mid X_{t}\right]+\frac{1}{2} \operatorname{Cov}\left[V_{T}+c \mid X_{t}\right]\right) \\
& =\exp \left(\gamma^{\prime}\left(e^{-\kappa(T-t)} X_{t}+\left(1-e^{-\kappa(T-t)}\right) \tilde{\mu}\right)+c+\frac{1}{2} \gamma^{\prime} \Sigma(t, T) \gamma\right) .
\end{aligned}
$$

Thus, we arrive at

$$
\begin{aligned}
\mathbb{E}\left[f\left(X_{T}\right) \mid X_{t}\right]= & \mathbb{E}\left[\cosh \left(V_{T}+c\right) \mid X_{t}\right] \\
= & \frac{1}{2}\left[\exp \left(\gamma^{\prime}\left(e^{-\kappa(T-t)} X_{t}+\left(1-e^{-\kappa(T-t)}\right) \tilde{\mu}\right)+c\right)\right. \\
& \left.+\exp \left(-\gamma^{\prime}\left(e^{-\kappa(T-t)} X_{t}+\left(1-e^{-\kappa(T-t)}\right) \tilde{\mu}\right)-c\right)\right] \exp \left(\frac{1}{2} \gamma^{\prime} \Sigma(t, T) \gamma\right) \\
= & \cosh \left(\mathbb{E}\left[V_{T} \mid X_{t}\right]+c\right) \exp \left(\frac{1}{2} \gamma^{\prime} \Sigma(t, T) \gamma\right)
\end{aligned}
$$

which yields the result.

From the zerobond price formula we can directly derive the nominal zerobond rates $y(t, T):=-\frac{1}{T-t} \log P(t, T)$ which are given in the next corollary:

Corollary 2.4. For the Hyperbolic Gaussian model, nominal zerobond rates $y(t, T)$ at time $t$ with expiry date $T$ are given by

$$
y(t, T)=\alpha-\frac{\log \cosh \left(\mathbb{E}\left[V_{T} \mid X_{t}\right]+c\right)}{T-t}+\frac{\log \cosh \left(V_{t}+c\right)}{T-t}-\frac{\gamma^{\prime} \Sigma(t, T) \gamma}{2(T-t)} .
$$

Instantaneous forward rates $f(t, T)=-\frac{\partial}{\partial T} \log (P(t, T))$ and shortrates $r_{t}=f(t, t)$ can be derived as well. In what follows $\|\cdot\|$ denotes the Euclidean norm.

Corollary 2.5. For the Hyperbolic Gaussian model, the shortrate is given by

$$
r_{t}=\alpha-\gamma^{\prime} \kappa\left(\tilde{\mu}-X_{t}\right) \tanh \left(V_{t}+c\right)-\frac{1}{2}\|\hat{C}\|^{2}
$$

and instantaneous forward rates $f(t, T)$ are given by

$$
f(t, T)=\alpha-\tanh \left(\mathbb{E}\left[V_{T} \mid X_{t}\right]+c\right) \gamma^{\prime} \kappa e^{-\kappa(T-t)}\left(X_{t}-\tilde{\mu}\right)+\gamma^{\prime} e^{-\kappa(T-t)} \rho e^{-\kappa(T-t)} \gamma
$$


Proof. By definition we obtain

$$
\begin{aligned}
& f(t, T)=-\frac{\partial}{\partial T} \log (P(t, T)) \\
= & -\frac{\partial}{\partial T} \log \left(e^{-\alpha(T-t)} \frac{\cosh \left(\mathbb{E}\left[V_{T} \mid X_{t}\right]+c\right)}{\cosh \left(V_{t}+c\right)} e^{\frac{1}{2} \gamma^{\prime} \Sigma(t, T) \gamma}\right) \\
= & -\frac{\partial}{\partial T}\left(-\alpha(T-t)+\log \left(\cosh \left(\mathbb{E}\left[V_{T} \mid X_{t}\right]+c\right)\right)+\frac{1}{2} \gamma^{\prime} \Sigma(t, T) \gamma\right) \\
= & \alpha-\tanh \left(\mathbb{E}\left[V_{T} \mid X_{t}\right]+c\right) \gamma^{\prime} \kappa e^{-\kappa(T-t)}\left(X_{t}-\tilde{\mu}\right)+\frac{1}{2} \gamma^{\prime} e^{-\kappa(T-t)} \rho e^{-\kappa(T-t)} \gamma
\end{aligned}
$$

and

$$
r_{t}=f(t, t)=\alpha-\tanh \left(V_{t}+c\right) \gamma^{\prime} \kappa\left(X_{t}-\tilde{\mu}\right)-\frac{1}{2}\|\hat{C}\|^{2}
$$

which yields the result.

We can see from the shortrate that the Hyperbolic Gaussian model allows for negative interest rates. Nevertheless, the probability of negative interest rates is small due to non-linearity of the function $f$ in the state process and the state process itself being conditionally normal. The higher $\alpha$, the lower the conditional probability of negative interest rates, so that we would prefer model estimates with high $\alpha$. However, $\alpha$ has a direct economic interpretation which might bound $\alpha$ and hence shape the probability of negative interest rates.

Theorem 2.6. The parameter $\alpha$ equals the asymptotic long rate $\lim _{T \rightarrow \infty} y(t, T)$.

Proof. Since $\lim _{T \rightarrow \infty} \mathbb{E}\left[X_{T} \mid X_{t}\right]=\tilde{\mu}$ and since log and cosh are continuous and cosh $\geq 1$ we obtain directly $\lim _{T \rightarrow \infty} y(t, T)=\alpha$.

The asymptotic long rate is very influential for long-term applications as it determines long-term discounting functions. As can be seen in Yao (1999), it is constant for many currently used term structure models, particularly also the affine model framework (see Duffie \& Kan (1996) and Dai \& Singleton (2000) $)$. Note that in most models the constant asymptotic long rate is a function of several model parameters, which makes sensitivity analysis with respect to the asymptotic long rate impossible, whereas the Hyperbolic Gaussian model allows for such sensitivity analysis. As the Hyperbolic Gaussian model also allows for an expansion to stock market dynamics, it is particularly interesting for long-term usage in pension or insurance applications which require long-term discounting.

Since the state price density in the Hyperbolic Gaussian model is in general not a supermartingale, it does not follow from standard literature that it is free of arbitrage. The proof of the following theorem can be found in the appendix.

Theorem 2.7. For the Hyperbolic Gaussian model as defined above, the bond market is free of arbitrage.

Remark 2.8. Note that the proof of Theorem 2.7 implies that the market price of risk is given by $\Lambda\left(V_{t}\right)=\tanh \left(V_{t}+c\right) \hat{C}$ and thus bounded. It can be used to define an equivalent martingale measure $\mathbb{Q}$ which does not depend on the time to maturity and to define a corresponding $\mathbb{Q}$ Brownian motion $\left(Z_{t}^{\mathbb{Q}}\right)$.

Finally note here that the Bond price formula in Theorem 2.3 is very easy to implement since it only requires the simulation of $X_{t}$ which is normally distributed. This is in contrast to formula (1.1) used by Cairns which is computationally demanding. Moreover, it has been shown in Pfeiffer (2010) that both models have similar properties (though Cairns model guarantees positive interest rates). Hence for practical purposes the Hyperbolic Gaussian model seems to be superior. 


\section{Joint Bond And Stock Market}

We extend now the Hyperbolic Gaussian bond model to include a stock. This is done by two different approaches: The dividend discount approach and a simple extension of the Black Scholes model.

3.1. The Dividend Discount Approach. In the dividend discount approach one assumes that the stock pays (random) dividends at deterministic, discrete time points $0 \leq \tau_{1}<\tau_{2}<\ldots$ with $\tau_{n} \rightarrow \infty$ for $n \rightarrow \infty$. More precisely we denote the dividend paid at time $\tau_{n}$ by $D_{\tau_{n}} \geq 0$. The stock can then be interpreted as a portfolio of infinitely many securities which pay $D_{\tau_{n}}$ at times $\tau_{n}$. Hence

$$
S_{t}=\sum_{\tau_{n} \geq t} \frac{\mathbb{E}\left[\varsigma_{\tau_{n}} D_{\tau_{n}} \mid \mathcal{F}_{t}\right]}{\varsigma_{t}} .
$$

This approach can be traced back to Gordon (1959). A continuous version in the state price density approach can be found in Graziano \& Rogers (2006).

Remark 3.1. It is important to note that due to dividend payments, we have to distinguish between the stock price and the wealth of a stock holder, which includes dividends already paid. For example the wealth of an investor at time $T$ who bought the stock at time $t$ is given by

$$
W_{T}=S_{T}+\sum_{t \leq \tau_{n} \leq T} \frac{\varsigma_{\tau_{n}}}{\varsigma_{T}} D_{\tau_{n}}
$$

For implementation of the joint stock and bond market model using the dividend discount approach, we have to specify the dividend payment process $\left(D_{t}\right)$ depending on the state process $\left(X_{t}\right)$ in such a way that the expectation $\mathbb{E}\left[\varsigma_{\tau_{n}} D_{\tau_{n}} \mid \mathcal{F}_{t}\right]$ in $(3.1)$ can be calculated in closed form. Furthermore, we require dividends to be non-negative and we implement an expected dividend growth over time to compensate for inflation, as company income from which dividends are to be paid should be inflation protected. An intuitive proposal defines

$$
D_{t}=D\left(t, X_{t}\right):=\exp \left(\bar{c}+\bar{\mu} t+\left(\gamma^{D}\right)^{\prime} X_{t}\right),
$$

for constants $\bar{c} \in \mathbb{R}, \bar{\mu} \in \mathbb{R}$ and $\gamma^{D} \in \mathbb{R}^{d}$. This specification guarantees positive dividends, varying around an exponential trend defined by $\bar{\mu}$. This dividend growth trend is introduced to capture the assumption of company income being adapted to rising prices. Unlike nominal bonds, stock holders therefore hold some protection against inflation implemented by the growth trend $\bar{\mu}$. The factor $\bar{c}$ is a multiplicative scaling factor for stock prices and dividends, which proved to be necessary in implementations but which is omitted in further theoretical considerations.

From an implementation point of view, it is important to note that our choice of $\left(D_{t}\right)$ allows for closed form solutions for the prices of dividend-paying securities, as the product of the two lognormally distributed variables $D_{t}$ and $\varsigma_{t}$ is again lognormally distributed. The current price $S_{t}^{n}$ of the stochastic dividend $D_{\tau_{n}}$ to be paid at time $\tau_{n}$ therefore is available in closed form by ${ }^{1}$

$$
\begin{aligned}
\varsigma_{t} S_{t}^{n}= & \mathbb{E}\left[\varsigma_{\tau_{n}} D_{\tau_{n}} \mid X_{t}\right] \\
= & \exp \left(-\alpha \tau_{n}+\bar{\mu} \tau_{n}+\left(\gamma^{D}\right)^{\prime} \mathbb{E}\left[X_{\tau_{n}} \mid X_{t}\right]+\frac{1}{2}\left(\gamma^{\prime} \Sigma\left(t, \tau_{n}\right) \gamma+\left(\gamma^{D}\right)^{\prime} \Sigma\left(t, \tau_{n}\right) \gamma^{D}\right)\right) \\
& \cosh \left(c+\gamma^{\prime} \mathbb{E}\left[X_{\tau_{n}} \mid X_{t}\right]+\gamma^{\prime} \Sigma\left(t, \tau_{n}\right) \gamma^{D}\right)
\end{aligned}
$$

which defines a stock price process by equation (3.1). We assume that the sum in (3.1) converges. This is e.g. the case when $\kappa_{i} \geq 0, \alpha \geq \bar{\mu}$ and $\tau_{n}=n$ which are realistic assumptions. It remains now to prove that the joint Hyperbolic Gaussian bond and stock market model is free of arbitrage. The proof of the next theorem can be found in the appendix.

\footnotetext{
${ }^{1}$ Note that the same definition of the dividend payment process may also be used to expand Rogers (1997) first and second examples which specify $f(x):=\exp x$ and $f(x):=\exp \left(c+x^{\prime} Q x\right)$, respectively.
} 
Theorem 3.2. For the Hyperbolic Gaussian model with the stock price process $\left(S_{t}\right)$ defined as the infinite sum of discounted dividends with payoffs given by $(3.2)$, the joint bond and stock market is free of arbitrage.

3.2. Extension of Black Scholes Model. Another simple possibility to obtain an arbitragefree joint bond and stock market is to use the classical Black Scholes model for the (non-dividend paying) stock and assume that under the risk neutral measure $\mathbb{Q}$ (cf. Remark 2.8), the drift of the stock is given by the shortrate of the Hyperbolic Gaussian model in Corollary 2.5, i.e.

$$
d S_{t}=S_{t}\left(r_{t} d t+\sigma^{T} C d Z_{t}^{\mathbb{Q}}\right) .
$$

By construction this yields an arbitrage-free market. An example for this approach may be found in Albrecht (2007), where a one-factor Vasicek model is used for the shortrate and a Black-Scholes model for the stock dynamics. Since the stock price is a stochastic exponential, it is also possible to derive an explicit formula for $\left(S_{t}\right)$.

\section{Estimation}

For estimation and risk-management, the physical measure is required. Whereas we are essentially free to specify the market price of risk and hence the physical measure, a standard approach derives the physical measure in such a way that the state process follows similar dynamics under both the physical measure and the measure typically used for pricing. For the Hyperbolic Gaussian model, this implies that the state process under the physical measure $\tilde{\mathbb{P}}$ should follow Ornstein-Uhlenbeck dynamics as under the pricing measure. Using Girsanov's theorem with

$$
\frac{d \tilde{\mathbb{P}}}{d \mathbb{P}}=\mathcal{E}\left(\int_{0}^{\cdot} \Lambda^{\tilde{\mathbb{P}}, \mathbb{P}}\left(X_{s}\right) d Z_{s}\right)
$$

where $\mathcal{E}$ is the stochastic exponential we know that

$$
d Z_{t}^{\tilde{\mathbb{P}}}=d Z_{t}+\Lambda^{\tilde{\mathbb{P}}, \mathbb{P}}\left(X_{t}\right) d t
$$

is a Brownian motion w.r.t. $\tilde{\mathbb{P}}$. Thus,

$$
\begin{aligned}
d X_{t} & =\kappa\left(\tilde{\mu}-X_{t}\right) d t+C d Z_{t} \\
& =\left(-C \Lambda^{\tilde{\mathbb{P}}, \mathbb{P}}\left(X_{t}\right)+\kappa \tilde{\mu}-\kappa X_{t}\right) d t+C d Z_{t}^{\tilde{\mathbb{P}}}
\end{aligned}
$$

implies

$$
\kappa \mu \stackrel{!}{=}-C \Lambda^{\tilde{\mathbb{P}}, \mathbb{P}}\left(X_{t}\right)+\kappa \tilde{\mu}
$$

so $\Lambda^{\tilde{\mathbb{P}}, \mathbb{P}}\left(X_{t}\right):=C^{-1} \kappa(\tilde{\mu}-\mu)$. As this drift correction term is constant, the Novikov condition is fulfilled and both measures are equivalent. Note that alternative specifications might provide better historical fit and in particular superior term premiums, see for example Duffee (2002).

4.1. Estimation by Extended Kalman Filter. We estimated both models with the extended Kalman filter. Since the approach is similar for the Black Scholes model, we restrict our presentation to the first model. A detailed description for the second model can be found in Pfeiffer (2010). The Hyperbolic Gaussian model allows for implementation of the extended Kalman filter for estimation of the model parameters in a Quasi-Maximum-Likelihood approach. The state process $\left(X_{t}\right)$ is the hidden process and the nominal zero bond rates $\left(Y_{t}\right)$ are observed for different maturities as well as the stock. For a general introduction to the Kalman filter, see e.g. Harvey (1991) or Kellerhals (2001). Using a discrete time grid and the standard notation 
$\mathcal{F}_{t}^{Y}=\sigma\left(\left\{Y_{0}, Y_{1}, \ldots, Y_{t}\right\}\right), t \in \mathbb{N}$ and

$$
\begin{aligned}
X_{t \mid t} & :=\mathbb{E}\left[X_{t} \mid \mathcal{F}_{t}^{Y}\right] \\
X_{t \mid t-1} & :=\mathbb{E}\left[X_{t} \mid \mathcal{F}_{t-1}^{Y}\right] \\
\Sigma_{t \mid t} & :=\mathbb{E}\left[\left(X_{t}-X_{t \mid t-1}\right)\left(X_{t}-X_{t \mid t-1}\right)^{\prime} \mid \mathcal{F}_{t}^{Y}\right] \\
\Sigma_{t \mid t-1} & :=\mathbb{E}\left[\left(X_{t}-X_{t \mid t-1}\right)\left(X_{t}-X_{t \mid t-1}\right)^{\prime} \mid \mathcal{F}_{t-1}^{Y}\right],
\end{aligned}
$$

the transition equations are given by

$$
X_{t \mid t-1}=e^{-\kappa} X_{t-1 \mid t-1}+\left(1-e^{-\kappa}\right) \mu,
$$

and

$$
\Sigma_{t \mid t-1}=e^{-\kappa} \Sigma_{t-1 \mid t-1} e^{-\kappa}+Q(\theta) .
$$

where $\theta$ denotes the vector of model parameters and the covariance matrix $Q(\theta)$ is given by (compare equation (2.8))

$$
Q(\theta)=\left(\frac{\rho_{l k}}{\kappa_{k}+\kappa_{l}}\left(1-e^{-\left(\kappa_{k}+\kappa_{l}\right)}\right)\right)_{l, k=1, \ldots, d} .
$$

The measurement equation is defined by

$$
Y_{t}=\left(\begin{array}{c}
y^{M}\left(t, t+\sigma_{1}\right) \\
\vdots \\
y^{M}\left(t, t+\sigma_{n}\right) \\
S^{M}(t)
\end{array}\right)=\left(\begin{array}{c}
g_{1}\left(X_{t} ; \theta\right) \\
\vdots \\
g_{n}\left(X_{t} ; \theta\right) \\
g_{n+1}\left(X_{t} ; \theta\right)
\end{array}\right)+\left(\begin{array}{c}
\epsilon_{t}^{(1)}(\theta) \\
\vdots \\
\epsilon_{t}^{(n)}(\theta) \\
\epsilon_{t}^{(n+1)}(\theta)
\end{array}\right)
$$

where $y^{M}\left(t, t+\sigma_{i}\right)$ is the interest rate observed in the market with time to maturity $\sigma_{i}, i=$ $1, \ldots, n$ and $S^{M}(t)$ is the observed stock price in the market at time $t$. The function $g_{i}, i=$ $1, \ldots, n$ is given by

$$
g_{i}\left(X_{t} ; \theta\right)=\alpha-\frac{\log \cosh \left(\gamma^{\prime} E\left[X_{t+\sigma_{i}} \mid X_{t}\right]\right)}{\sigma_{i}}+\frac{\log \cosh \left(\gamma^{\prime} X_{t}+c\right)}{\sigma_{i}}-\frac{\gamma^{\prime} \Sigma\left(t, t+\sigma_{i}\right) \gamma}{\sigma_{i}} .
$$

The function $g_{n+1}$ describes the model-implied stock price and is given by

$$
\begin{aligned}
g_{n+1}\left(X_{t} ; \theta\right)= & \sum_{\tau_{n}>t} \frac{1}{2 \varsigma_{t}} \exp \left(-\alpha \tau_{n}+\bar{\mu} \tau_{n}\right)\left[\operatorname { e x p } \left(\bar{c}+\left(\gamma^{D}+\gamma\right)^{\prime} \mathbb{E}\left[X_{\tau_{n}} \mid X_{t}\right]\right.\right. \\
& \left.+\frac{1}{2}\left(\gamma^{D}+\gamma\right)^{\prime} \Sigma\left(t, \tau_{n}\right)\left(\gamma^{D}+\gamma\right)\right) \\
& \left.+\exp \left(-\bar{c}+\left(\gamma^{D}-\gamma\right)^{\prime} \mathbb{E}\left[X_{\tau_{n}} \mid X_{t}\right]+\frac{1}{2}\left(\gamma^{D}-\gamma\right)^{\prime} \Sigma\left(t, \tau_{n}\right)\left(\gamma^{D}-\gamma\right)\right)\right] .
\end{aligned}
$$

Furthermore $\epsilon_{t}(\theta)=\left(\epsilon_{t}^{(1)}(\theta), \ldots, \epsilon_{t}^{(n+1)}(\theta)\right) \in \mathbb{R}^{(n+1)}$ is a multivariate normal error term with covariance matrix

$$
\operatorname{Cov}\left(\epsilon_{t}\right):=H_{t}=\operatorname{diag}\left(\nu, \ldots, \nu, \nu^{S}\right) \in \mathbb{R}^{(n+1) \times(n+1)}
$$

where $\nu$ is used for the measurement errors of yields and $\nu^{S}$ for the measurement errors of the stock price. This specification is due to the scaling difference between interest rates, roughly varying between 0 and 0.2 , and stock prices roughly varying between 100 and 1500 . Individual measurement errors for each maturity may allow for further insights, yet this benefit is made up for by the substantially higher number of model parameters one had to estimate in this case. For the updating step, we require the Kalman gain matrix

$$
K_{t}:=\Sigma_{t \mid t-1} B_{t \mid t-1}^{\prime} F_{t \mid t-1}^{-1}
$$


where $B_{t \mid t-1}$ is the Jacobi matrix of the non-linear measurement function $g=\left(g_{1}, \ldots, g_{n+1}\right)$ of the Hyperbolic Gaussian model given by

$$
B_{t \mid t-1}=\left.\left(\begin{array}{ccc}
\frac{\partial}{\partial x_{1}} g_{1}(x, \theta) & \cdots & \frac{\partial}{\partial x_{d}} g_{1}(x ; \theta) \\
\vdots & & \vdots \\
\frac{\partial}{\partial x_{1}} g_{n}(x ; \theta) & \cdots & \frac{\partial}{\partial x_{d}} g_{n}(x ; \theta) \\
\frac{\partial}{\partial x_{1}} g_{n+1}(x ; \theta) & \cdots & \frac{\partial}{\partial x_{d}} g_{n+1}(x ; \theta)
\end{array}\right)\right|_{x=X_{t \mid t-1}} \in \mathbb{R}^{(n+1) \times d} .
$$

Note that $\theta=\left(\gamma, \gamma^{D}, \mu, \bar{\mu}, \kappa, \rho, c, \nu, \nu^{S}\right)$. The required derivatives for the yield and stock price measurements can be derived using

$$
\frac{\partial}{\partial x_{i}} P(t, T)=\gamma_{i} P(t, T)\left[\tanh \left(\gamma^{\prime} \mathbb{E}\left[X_{T} \mid X_{t}\right]+c\right) e^{-\kappa_{i} t}-\tanh \left(\gamma^{\prime} X_{t}+c\right)\right]
$$

and

$$
\frac{\partial}{\partial x_{i}} S_{t}=S_{t}\left(\gamma_{i}^{D} e^{-\kappa_{i}(T-t)}+\tanh \left(h\left(t, X_{t}\right)\right) \gamma_{i} e^{-\kappa_{i}(T-t)}-\gamma_{i} \tanh \left(\gamma^{\prime} X_{t}+c\right)\right)
$$

respectively with $h\left(t, X_{t}\right)=\mathbb{E}\left[V_{T} \mid X_{t}\right]+\gamma^{\prime} \Sigma(t, T) \gamma^{D}+c$. With $F_{t \mid t-1}=B_{t \mid t-1} \Sigma_{t \mid t-1} B_{t \mid t-1}^{\prime}+H_{t}$, the Kalman gain matrix can be calculated. The prediction error

$$
v_{t}=\left(y^{M}\left(t, t+\sigma_{1}\right), \ldots, y^{M}\left(t, t+\sigma_{n}\right), S_{t}^{M}\right)^{\prime}-g\left(X_{t \mid t-1}, \theta\right) .
$$

yields the updating steps

$$
X_{t \mid t}=X_{t \mid t-1}+K_{t} v_{t}
$$

and

$$
\Sigma_{t \mid t}=\Sigma_{t \mid t-1}-K_{t} B_{t \mid t-1} \Sigma_{t \mid t-1}
$$

which concludes the filter.

For implementation, we have to cut off the infinite sum of dividend paying securities for computational reasons. As the dividends are non-negative, even for lower interest rates an additional $(n+1)$-th dividend likely increases the stock price. Note that a positive dividend growth trend $\bar{\mu}$ may compensate the discounting of future dividends, thus the current value of expected future dividends will not necessarily decrease with higher times to maturity. In general, we expect that cutting off dividend payments of later dates implies that early dividends will be overestimated to make up for omitted dividends.

Not surprisingly, we found a conflicting role of the asymptotic long rate $\alpha$ determining longterm discounting, and the dividend growth rate $\bar{\mu}$. In the joint model, estimates of both these parameters were very unstable. Restricting $\alpha=0.042$ in our estimates resulted in better stability of both parameters. The economic justification of the specification $\alpha=0.042$ is based on a simplifying application of the Fisher hypothesis (Fisher $(1930))$, which partitions nominal interest rates into the expected inflation rate and a real rate. Now the long-term average of the real rate is taken as $2.2 \%$ and the expected asymptotic inflation rate is interpreted as the inflation target of the central bank, currently $2 \%$. The Fisher hypothesis implies that the sum of both equals the nominal interest rate. Using historical dividend data should solve these problems, yet due to rather small dividend payments in absolute terms the cut-off levels are necessarily high, implying a higher computational effort for estimation. Furthermore, at least in case of US data, each time step would require implementation of an individual dividend payment schedule as the times between dividend payments are not necessarily constant. Finally, we want to use stock index data, motivated by our proposition of the Hyperbolic Gaussian model for long-term insurance applications, and the problems of irregular dividend payments are even worse for an index or generally for a portfolio of stocks. Therefore, we omitted the inclusion of historical dividend data. Effectively, we model a theoretical finite cash flow whose current value equals the stock price. Omitting historical dividend payments allows for simplifying assumptions regarding 


\begin{tabular}{|c|c|c|c|c|c|c|c|c|}
\hline $0.25 \mathrm{y}$ & $0.5 \mathrm{y}$ & $1 \mathrm{y}$ & $2 \mathrm{y}$ & $3 \mathrm{y}$ & $5 \mathrm{y}$ & $7 \mathrm{y}$ & $10 \mathrm{y}$ & $S \& P 500$ \\
\hline 13.7 & 5.0 & 8.6 & 13.3 & 9.2 & 5.9 & 5.5 & 9.9 & 0.7 \\
13.8 & 5.2 & 8.7 & 13.3 & 9.6 & 5.6 & 5.5 & 9.7 & 0.9 \\
13.4 & 5.4 & 9.0 & 12.7 & 9.9 & 6.0 & 6.0 & 10.7 & 0.8 \\
\hline
\end{tabular}

TABLE 1. Historical mean absolute errors in basis points for the yields and in ticks for the index.

frequency and regularity of dividend payment dates. We deem these simplifying assumptions not less viable than the frequent assumption of a continuous dividend yield on stocks.

The components of the state process are assumed to be Ornstein-Uhlenbeck processes. These are typically easy to be estimated, unless mean reversion is low. In that case, the (extended) Kalman filter may be unable to derive the true value of long-term mean $\mu$, as $\mu$ enters the filter equations only through the transition equation (4.1) and the impact of $\mu$ in this equation decreases with the mean reversion factor $\kappa$. Consequently, one has to examine the filtered state process to assess the fit of $\mu$.

In the following estimates, we restricted the long-term mean of the state process under the pricing measure to be zero, $\tilde{\mu}=0$. The extended Kalman filter is able to estimate non-restricted $\tilde{\mu}$ in case of the pure bond market model, although improvement of the more general model is negligible. In the joint bond and stock market model, restricting $\tilde{\mu}$ improved estimation speed and stability substantially.

4.2. Estimation-Results. For estimation of interest rates, we use a three-dimensional state process and a dataset of the Federal Reserve consisting of $0.25-, 0.5-, 1-, 2-, 3-, 5-, 7-$ and 10-year yields derived from US Treasury securities from January 1984 to January 2008. The dataset is obtained from the Federal Reserve download portal. For estimation of the stock market we use $S \& P 500$ price index data. This reflects well the assumption that the insurance company invests in a well diversified stock fund rather than a single stock. Furthermore, there is no dividend jump in the data.

4.3. Results for the Dividend Discount Approach. For simplicity, we did not use dividend data directly, but assume that the dividend payment process is semiannually and unobservable. In practice, we are replicating a dividend payment process which implies the same price data as the $S \& P 500$ index.

We found that the extended Kalman filter very quickly provides parameter estimates which closely fit historical interest rates and the dynamics of the stock price, but only a few of those provide a close fit of the absolute stock price as well. Typically, the model-implied stock price is highly correlated to the observed stock price, yet differs in absolute value. To overcome this drawback, a second estimation step was implemented which uses these first estimates and fits only the parameters describing the stock price dynamics, that is $\gamma^{D}, \rho_{i j}, \bar{\mu}$ and $c$, to the stock price observations. The final results differ from the initial values only slightly in Loglikelihood, yet guarantee in most cases a good historical fit of the stock price (see Figure 1). As the extended Kalman filter provides only Quasi-ML estimates, we deem such a correcting approach as viable. Table 2 provides three estimated parameter sets. We see that the parameter estimates still show considerable variability, which may be due to overparametrization. Note that multiple local maxima of the Loglikelihood function are a typical finding in complex applications. However note that the measurement errors $\nu$ and $\nu^{S}$ for the stock are very small. Table 1 provides absolute historical errors of the model. We see that for all estimates historical errors are extremely small, in particular regarding the stock, and very similar across different estimated parameter sets.

We examine the filtered underlying state vector in figure 2. We find clear correlation between one of the state factor components and the slope of the interest curve and a second state factor component and the stock price. The third state factor is correlated to the 10-year rate. Both 


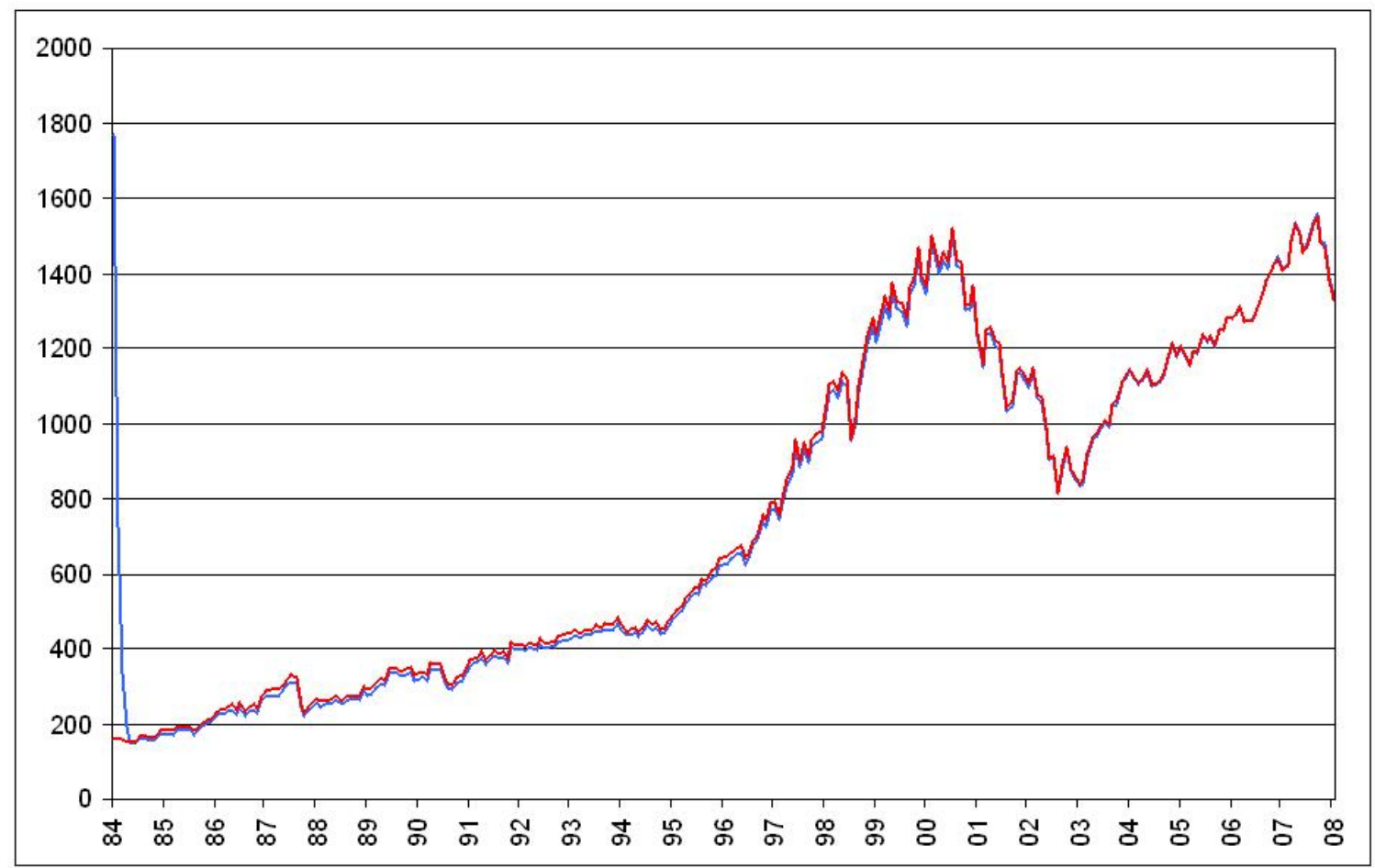

Figure 1. Model implied (blue) and historical $S \& P 500$ prices in the dividend discount model.

\begin{tabular}{|c|c|c|c|c|c|c|c|c|c|c|c|c|}
\hline \hline$\gamma_{1}$ & $\gamma_{2}$ & $\gamma_{3}$ & $\gamma_{1}^{D}$ & $\gamma_{2}^{D}$ & $\gamma_{3}^{D}$ & $\mu_{1}$ & $\mu_{2}$ & $\mu_{3}$ & $\bar{\mu}$ & $\kappa_{1}$ & $\kappa_{2}$ & $\kappa_{3}$ \\
\hline \hline-0.47 & -0.07 & 0.44 & -0.24 & -0.24 & 0.56 & 0 & 0.1 & 1.7 & 0 & 0.067 & 0.343 & 0.063 \\
-0.02 & 0.03 & 0.23 & 0.23 & -0.04 & 0.01 & 15.7 & 0.7 & 1.2 & 0 & 0.116 & 0.432 & 0.052 \\
0.20 & 0.02 & -0.16 & 0.72 & -0.80 & 0.01 & 5.8 & -0.6 & 1.8 & 0 & 0.012 & 0.473 & 0.058 \\
\hline \hline$\rho_{12}$ & $\rho_{13}$ & $\rho_{23}$ & $c$ & $\nu$ & $\nu^{S}$ & LogL & & & & & & \\
\hline \hline-0.70 & 0.70 & -0.05 & 0 & 0.0013 & 0.0008 & 12205 & & & & & & \\
-0.01 & -0.01 & -0.47 & -0.3 & 0.0013 & 0.0211 & 12283 & & & & & & \\
-0.32 & -0.53 & 0.69 & -13.4 & 0.0013 & 0.0274 & 12270 & & & & & & \\
\hline \hline
\end{tabular}

TABle 2. Estimates by the extended Kalman filter for the Hyperbolic Gaussian model using US Treasury term structure data and S\&P500 stock market data from January 1984 to January 2008.

the dynamics of the level and the stock price factor provide very small mean reversion, as could be seen in our estimates of $\mu_{2}$ and $\mu_{3}$.

4.4. Results for Black Scholes model. We implemented again a three-factor model. One main difference between the Black-Scholes based approach and the dividend discount model is computational speed. Whereas the dividend discount model is computationally slow, the Black-Scholes based approach is very fast in estimation and simulation alike, since calculation of the current stock return is computationally equivalent to computation of an interest rate with given maturity. First note that stock returns differ substantially from interest rates in autocorrelation and variance. To account for these differences, we implemented two approaches, based on restrictions of the parameter vector $\gamma$. The framework presented by Albrecht (2007) implies a pure stock market factor, which in our model would be equivalent to $\gamma_{3}=0$. We consider two model frameworks, one without restrictions on $\gamma$, which implies that the state vector drives both stock and bond markets, and one with $\gamma_{3}=0$, which implies two state vector 

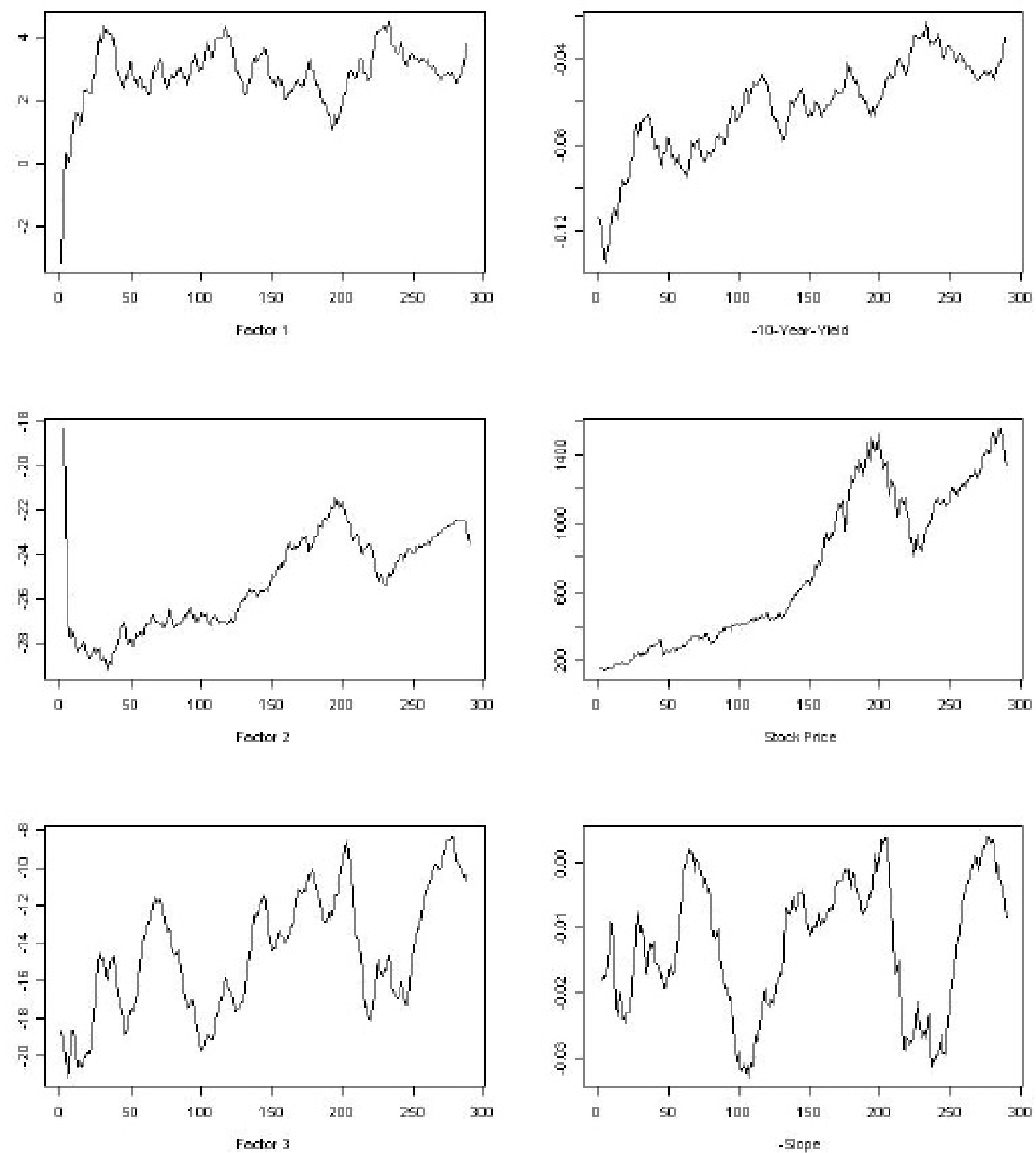

FiguRE 2. Filtered state vector components (left) and empirical proxies of the first principal components of the term structure.

components driving bond and stock markets, and one state vector component driving only the stock market. Note that implementation of these restrictions is very easy, and pricing formulae still hold.

Table 3 provides MAEs of implied yield curves. We see that restricting $\gamma$ implies a poorer term structure fit. The reason is that all three vector components contain term structure data for general $\gamma$, whereas $\gamma_{3}=0$ guarantees that the third state vector component drives stock returns only and therefore improves stock return fit. Considering historical fit of the stock price, we have MAEs in ticks of more than 1700 for general $\gamma$ and 18 basis points for $\gamma_{3}=0$. The reason is that for $\gamma_{3}=0$, the Extended Kalman filter fits $Z_{3}$ to the observed stock price, whereas with general $\gamma$ a trade-off exists between fitting the stock price and the term structure. Given the lower Loglikelihood values of the restricted approach, we can expect that the distribution of 


\begin{tabular}{|c|c|c|c|c|c|c|c|}
\hline \multicolumn{8}{|c|}{ Assuming $\gamma_{3} \neq 0$} \\
\hline $0.25 \mathrm{y}$ & $0.5 y$ & $1 \mathrm{y}$ & $2 y$ & $3 y$ & $5 y$ & $7 y$ & $10 y$ \\
\hline 5.3 & 4.4 & 5.8 & 5.3 & 4.0 & 3.5 & 4.5 & 5.9 \\
\hline 5.4 & 4.4 & 5.8 & 5.4 & 4.1 & 3.6 & 4.5 & 5.9 \\
\hline 5.4 & 4.4 & 5.8 & 5.4 & 4.0 & 3.5 & 4.5 & 5.9 \\
\hline \multicolumn{8}{|c|}{ Assuming $\gamma_{3}=0$} \\
\hline 12.7 & 5.2 & 8.8 & 12.5 & 8.8 & 6.1 & 5.4 & 9.9 \\
\hline 12.7 & 5.2 & 8.7 & 12.5 & 8.8 & 6.0 & 5.4 & 9.9 \\
\hline 12.7 & 5.2 & 8.8 & 12.5 & 8.8 & 6.0 & 5.4 & 9.8 \\
\hline
\end{tabular}

TABLE 3. Historical mean absolute errors in basis points for the yields.

$Z_{3}$ according to the filtering in the restricted case deviates from the theoretical model-implied distribution of $Z_{3}$. By definition of the model, stock returns are normally distributed, whereas it is well known that this is not the case in reality. Therefore the good historical fit of the model assuming $\gamma_{3}=0$ does not reflect the basic problems this approach takes from the Black-Scholes model. We expect that non-normality of stock returns is responsible for the lower Loglikelihood values of the restricted model. A straightforward improvement of the joint model would allow for stochastic volatility of the state vectors, thereby introducing stochastic volatility in the stock market as well as the bond market.

4.5. Comparison. In general, stock market models may be implemented using return-based or price-based approaches. Both approaches have their merits: banking applications typically consider stock derivatives, which are based on stock prices rather than returns. Therefore, pricebased approaches are superior for banking applications. Once dividend payments are introduced, however, the situation changes. To realistically implement discrete dividend payments, we require path-dependent approaches and consider reinvestment of dividends payed. In insurance applications, reinvestment of dividends is an important task since, over the long run, dividend returns make up a sizeable part of overall stock returns and furthermore intermediate dividend payments provide free cash flows without the need to liquidate assets under management. The Black Scholes model is computationally superior to the dividend discount model, yet it does not fit historical data to the same extent as the dividend discount model.

\section{CONCLUSiON}

The Hyperbolic Gaussian model is based on the state price density approach and allows for an easy estimation of parameters and fast Monte Carlo simulation. It can also be extended to a joint bond and stock market. Based upon its good long-term fit of both stock prices and interest rates and the increased importance of changes in interest rates, the Hyperbolic Gaussian model might provide a useful tool for long-term risk management like DFA due to the simple and fast implementation of Monte Carlo simulations. It therefore could be useful for insurance applications.

\section{Appendix}

The proof of Theorem 2.7 is as follows:

Proof. Using the Itô-Doeblin formula it is easy to see that the state price density

$$
\varsigma_{t}=\frac{1}{2} e^{-\alpha t}\left(e^{V_{t}+c}+e^{-V_{t}-c}\right)
$$

satisfies the SDE

$$
d \varsigma_{t}=-\alpha \varsigma_{t} d t+\tanh \left(V_{t}+c\right) \varsigma_{t} d V_{t}+\frac{1}{2} \varsigma_{t} d<V>_{t}
$$


Since $<V>_{t}=\|\hat{C}\|^{2} t$ we obtain

$$
d \varsigma_{t}=\varsigma_{t}\left(-\alpha+\gamma^{\prime} \kappa\left(\tilde{\mu}-X_{t}\right) \tanh \left(V_{t}+c\right)+\frac{1}{2} \hat{C}^{\prime} \hat{C}\right) d t+\varsigma_{t} \tanh \left(V_{t}+c\right) \hat{C} d Z_{t}
$$

In view of Corollary 2.5 we obtain

$$
d \varsigma_{t}=-\varsigma_{t} r_{t} d t+\varsigma_{t} \tanh \left(V_{t}+c\right) \hat{C} d Z_{t} .
$$

Let us denote by $\mathcal{E}(X)$ the stochastic exponential of the process $X$. Since $\int_{0}^{\cdot} r_{s} d s$ is of bounded variation we obtain

$$
\begin{aligned}
\varsigma_{t} & =\mathcal{E}\left(\int_{0} r_{s} d s\right)_{t} \cdot \mathcal{E}\left(\int_{0}^{\cdot} \tanh \left(V_{s}+c\right) \hat{C} d Z_{s}\right)_{t} \\
& =\exp \left(-\int_{0}^{t} r_{s} d s\right) \cdot \mathcal{E}\left(\int_{0}^{\cdot} \tanh \left(V_{s}+c\right) \hat{C} d Z_{s}\right)_{t} .
\end{aligned}
$$

If we define $B_{t}=\exp \left(\int_{0}^{t} r_{s} d s\right)$ and $L_{t}:=\mathcal{E}\left(\int_{0}^{\cdot} \tanh \left(V_{s}+c\right) \hat{C} d Z_{s}\right)_{t}$ we obtain $\varsigma_{t}=B_{t}^{-1} L_{t}$. Note that since tanh is bounded, $\left(L_{t}\right)$ is an $\left(\mathcal{F}_{t}\right)$-martingale with expectation 1 . Hence we can define the probability measure $\mathbb{Q}$ by $\left.\frac{d \mathbb{Q}}{d \mathbb{P}}\right|_{\mathcal{F}_{t}}=L_{t}$. Note that $\mathbb{Q}$ does not depend on $T$. From the Bayes formula we obtain

$$
P(t, T)=\frac{\mathbb{E}\left[\varsigma_{T} \mid \mathcal{F}_{t}\right]}{\varsigma_{t}}=\frac{\mathbb{E}\left[B_{T}^{-1} L_{T} \mid \mathcal{F}_{t}\right]}{B_{t}^{-1} L_{t}}=B_{t} \mathbb{E}_{\mathbb{Q}}\left[B_{T}^{-1} \mid F_{t}\right] .
$$

Hence the discounted bond price is a martingale under $\mathbb{Q}$ which shows that the market is free of arbitrage.

The proof of Theorem 3.2 is as follows:

Proof. It is sufficient to show that $\varsigma^{-1} B_{t}^{-1} \mathbb{E}\left[\varsigma_{\tau_{n}} D_{\tau_{n}} \mid \mathcal{F}_{t}\right]$ is a $\mathbb{Q}$-martingale for the same $\mathbb{Q}$ as in the proof of Theorem 3.2. However, the Bayes formula again implies

$$
\begin{aligned}
\frac{\mathbb{E}\left[\varsigma_{\tau_{n}} D_{\tau_{n}} \mid \mathcal{F}_{t}\right]}{\varsigma_{t}} & =\frac{\mathbb{E}\left[B_{\tau_{n}}^{-1} L_{\tau_{n}} D_{\tau_{n}} \mid \mathcal{F}_{t}\right]}{B_{t}^{-1} L_{t}} \\
& =B_{t} \mathbb{E}_{\mathbb{Q}}\left[B_{\tau_{n}}^{-1} D_{\tau_{n}} \mid \mathcal{F}_{t}\right]
\end{aligned}
$$

which implies the statement.

\section{REFERENCES}

Albrecht, P. (2007). Einige Überlegungen zur simultanen Modellierung von Aktienindex und Zinsstruktur. Mannheimer Manuskripte zu Risikotheorie, Portfolio Management und Versicherungswirtschaft .

Brigo, D. \& Mercurio, F. (2001). Interest Rate Models: Theory and Practice. Springer.

Cairns, A. J. G. (2004). A family of term-structure models for long-term risk management and derivative pricing. Mathematical Finance 14, 415-444.

Cairns, A. J. G. (2008). Interest Rate Models. Princeton University Press, Princeton, NJ.

Dai, Q. \& Singleton, K. J. (2000). Specification analysis of affine term structure models. Journal of Finance 55, 1943-1978.

Duffee, G. R. (2002). Term premia and interest rate forecasts in affine models. Journal of Finance $\mathbf{5 7}, 405-443$.

Duffie, D. (1992). Dynamic Asset Pricing. Princeton University Press, Princeton, NJ.

Duffie, D. \& Kan, R. (1996). A yield-factor model of interest rates. Mathematical Finance 6, 379-406.

Filipovic, D. (2009). Term-Structure Models. Springer.

Fisher, I. (1930). The Theory of Interest. The Macmillan Co., New York.

Flesaker, B. \& Hughston, L. (1996). Positive interest. Risk 46-49.

Gordon, M. (1959). Dividends, earnings, and stock prices. The Review of Economics and Statistics 41, 99-105. 
Graziano, G. D. \& Rogers, L. (2006). Hybrid derivatives pricing under the potential approach. Working paper, University of Cambridge.

Harvey, A. C. (1991). Forecasting, Structural Time Series Models and the Kalman Filter. Cambridge Books, Cambridge University Press.

Kellerhals, B. P. (2001). Financial Pricing Models in Continuous Time and Kalman Filtering. Lecture notes in economics and mathematical systems, Springer, Berlin.

Litterman, R. \& Scheinkman, J. (1991). Common factors affecting bond returns. Journal of Fixed Income 62-74.

Musiela, M. \& Rutkowski, M. (2005). Martingale Methods in Financial Modeling, vol. 36 of Stochastic Modelling and Applied Probability. 2 ed., Springer.

Pfeiffer, R. (2010). State price density models for the term structure of interest rates. applications to insurance and expansions to the stock market macroeconomic variables. http: //www.digbib.ubka.uni-karlsruhe.de/volltexte/1000019585. Ph.D. thesis, KIT.

Rebonato, R. (2002). Modern Pricing of Interest-Rate Derivatives: The Libor Market Model and Beyond. Princeton University Press.

Rogers, L. (1997). The potential approach to the term structure of interest rates and foreign exchange rates. Journal of Mathematical Finance 7, 157-176.

Rutkowski, M. (1997). A note on the Flesaker-Hughston model of the term structure of interest rates. Applied Mathematical Finance 151-163.

Wilkie, A. (1984). Steps towards a comprehensive stochastic model. Research discussion paper, The Institute of Actuaries.

Wilkie, A. (1986). A stochastic investment model for actuarial use. Transactions of the Faculty of Actuaries 39, 341-403.

Wilkie, A. (1995). More on a stochastic asset model for actuarial use. British Actuarial Journal 1, 777-964.

Yao, Y. (1999). Term structure modeling and asymptotic long rate. Insurance: Mathematics and Economics 25, 327-336.

Yao, Y. (2001). State price density, Esscher transforms, and pricing options on stocks, bonds, and foreign exchange rates. North American Actuarial Journal 5, 104-117. 